\title{
DEPDC1 drives hepatocellular carcinoma cell proliferation, invasion and angiogenesis by regulating the CCL20/CCR6 signaling pathway
}

\author{
WUBIN GUO ${ }^{1,2}$, HUI LI ${ }^{1}$, HUAN LIU ${ }^{3}$, XIN MA $^{2}$, SIJIN YANG ${ }^{4}$ and ZIWEI WANG ${ }^{1}$ \\ ${ }^{1}$ Department of Gastrointestinal Surgery, The First Affiliated Hospital of Chongqing Medical University, Chongqing 400016; \\ ${ }^{2}$ Department of General Surgery, ${ }^{3}$ Research Laboratory of Biomedical Engineering and ${ }^{4}$ Department of Cardiovascular Medicine, \\ The TCM Affiliated Hospital of Southwest Medical University, Luzhou, Sichuan 646000, P.R. China
}

Received January 14, 2019; Accepted July 3, 2019

DOI: 10.3892/or.2019.7221

\begin{abstract}
DEP domain containing 1 (DEPDC1) functions as an oncogene in hepatocellular carcinoma (HCC). However, the underlying mechanism of DEPDC1 remains largely unknown. The present study revealed that DEPDC1 knockdown inhibited HCC cell proliferation, colony formation and invasion in vitro and suppressed the growth of HCC xenografts in vivo. Furthermore, DEPDC1 overexpression promoted HCC cell proliferation, colony formation and invasion. DNA microarray, reverse transcription-quantitative-PCR and western blotting results demonstrated that DEPDC1 knockdown in Huh-7 significantly inhibited the expression of chemokine (C-C motif) ligand 20 (CCL20) and chemokine (C-C motif) receptor 6 (CCR6). In addition, the expression of CCL20 and CCR6 were upregulated in HCC tissues and cell lines, and were positively correlated with DEPDC1 expression. CCL20 or CCR6 knockdown via small interfering RNA reversed the effects of DEPDC1 overexpression in HCC cells. Furthermore, it was revealed that conditioned medium from DEPDC1 upregulated $\mathrm{Li}-7$ and $\mathrm{Hep} 3 \mathrm{~B}$ cells led to angiogenesis in vitro, whereas CCL20 knockdown in Li-7 and Hep3B cells or CCR6 knockdown in human umbilical vein endothelial cells reversed the angiogenic effect of DEPDC1 overexpression. In conclusion, DEPDC1 facilitated cell proliferation, invasion and angiogenesis via the CCL20/CCR6 pathway in HCC.
\end{abstract}

Correspondence to: Professor Ziwei Wang, Department of Gastrointestinal Surgery, The First Affiliated Hospital of Chongqing Medical University, 1 Firendship Road, Chongqing 400016, P.R. China E-mail: wangziwei@hospital.cqmu.edu.cn

Professor Sijin Yang, Department of Cardiovascular Medicine, The TCM Affiliated Hospital of Southwest Medical University, 182 Chunhui Road, Luzhou, Sichuan 646000, P.R. China

E-mail: ysjimn@swmu.edu.cn

Key words: DEP domain containing 1, chemokine ligand 20, chemokine receptor 6 , proliferation, invasion, angiogenesis

\section{Introduction}

Hepatocellular carcinoma (HCC) is a highly lethal cancer with an increasing incidence, making it a cause of major health problems worldwide $(1,2)$. Recently, surgical treatments including hepatic resection, liver transplantation, local ablative therapy and transarterial chemoembolization have been the most successful approaches to HCC. Patients with $\mathrm{HCC}$ have benefitted from significant improvements in tumor identification and patient survival $(3,4)$. However, the incidence rate of HCC is almost equivalent to its mortality rate in most countries, indicating a lack of early diagnostic methods and effective therapies (5). Therefore, studies that identify novel factors involved in HCC oncogenesis are essential for the improvement of early diagnosis and therapeutic approaches.

The DEP domain containing 1 (DEPDC1) gene is a highly conserved protein that is located at $1 \mathrm{p} 31.3$. It is primarily expressed in the testis and is not detected in other normal human tissues (6). Recently, several studies revealed that DEPDC1 was upregulated in many tumor types, indicating its important role in tumorigenesis (7-10). For example, DEPDC1 was upregulated in bladder carcinogenesis and the inhibition of DEPDC1 via small-interfering RNA (siRNA) significantly suppressed the growth of bladder cancer cells (7). In nasopharyngeal carcinoma, DEPDC1 was overexpressed in tumor tissues and DEPDC1 depletion induced the inhibition of cell proliferation, migration and invasion (9). Thus, DEPDC1 usually functions as an oncogene. In addition, HCC has been determined to overexpress DEPDC1, which is associated with a poor prognosis (11). Furthermore, a previous study revealed that disrupting the DEPDC1-ZNF224 complex via the 1 R-DEP peptide induced apoptosis and inhibited the proliferation of HepG2 cells (12). Qu et al (13) demonstrated that DEPDC1 upregulation induced HCC cell proliferation and neoplasm metastasis. The present study revealed that DEPDC1 knockdown significantly suppressed HCC cell proliferation, colony formation and invasion, and DEPDC1 overexpression significantly induced HCC cell proliferation, colony formation and invasion. However, the role and precise mechanism of DEPDC1 in HCC is still not fully understood. 
The aim of the present study was to elucidate the underlying mechanism of DEPDC1 in HCC. After a DNA microarray assay was performed, Gene ontology (GO) annotation results revealed that DEPDC1 was involved in vasculature development and blood vessel development. Furthermore, Kyoto Encyclopedia of Genes and Genomes (KEGG) pathway analysis revealed cytokine-cytokine receptor interactions were significantly enriched. DNA microarray, reverse transcription-quantitative PCR (RT-qPCR) and western blotting results revealed that DEPDC1 knockdown significantly suppressed the expression of chemokine (C-C motif) ligand 20 (CCL20) and chemokine (C-C motif) receptor 6 (CCR6). Recently, the CCL20/CCR6 axis has been determined to be involved in HCC cell growth and invasion (14,15). Additionally, Benkheil et al (16) revealed that the CCL20/CCR6 axis contributed to hepatic angiogenesis in hepatitis C virus (HCV)-associated HCC. Angiogenesis is vital for the growth of cancer and the development of metastasis (17). Thus, the CCL20/CCR6 axis may serve an important role in DEPDC1-mediated HCC progression. Based on the aforementioned hypothesis, the present study further investigated the role of the CCL20/CCR6 axis in DEPDC1-mediated HCC progression, which may elucidate a novel mechanism of DEPDC1 in HCC.

\section{Materials and methods}

Ethics statement. The present was approved by the Ethics Committee of the First Affiliated Hospital of Chongqing Medical University (Chongqing, China). All animal experiments were performed as indicated in the Guidelines of the National Institutes of Health for Animal Care (Guide for the Care and Use of Laboratory Animals, Department of Health and Human Services, NIH publication no. 86-23, revised 1985).

Human tissues. A total of 12 pairs of tumor tissue with matched adjacent normal tissue were obtained from patients diagnosed with $\mathrm{HCC}$ at the First Affiliated Hospital of Chongqing Medical University (Chongqing, China) between October 2016 and July 2017. The patients comprised of 10 men and 2 women from 45 to 73 years of age. All patients provided their written informed consent. None of the patients had received radiotherapy, immunotherapy or chemotherapy prior to surgery. All tissue samples were frozen in liquid nitrogen and subsequently stored at $-80^{\circ} \mathrm{C}$ for RT-qPCR analysis.

Immunohistochemistry (IHC). IHC examination of DEPDC1, CCL20 and CCR6 was performed as previously described (18). HCC tissues embedded in paraffin were cut into 4- $\mu$ m-thick sections. Sections were then subjected to dewaxing and rehydration, after which antigen retrieval was performed via microwave treatment for $15 \mathrm{~min}$. Samples were subsequently treated with $3 \%$ hydrogen peroxide for $15 \mathrm{~min}$ to block endogenous peroxidase activity and incubated with $10 \%$ goat non-immune serum for $30 \mathrm{~min}$. Sections were incubated with the following antibodies overnight at $4^{\circ} \mathrm{C}$ : Rabbit anti-human DEPDC1 (1:50; cat. no. GTX17614; GeneTex, Inc.), rabbit anti-human CCL20 (1:200; cat. no. 26527-1-AP; ProteinTech Group, Inc.) and rabbit anti-human CCR6 (1:1,000; cat. no. ab227036; Abcam). Sections were then incubated with corresponding goat anti-rabbit secondary antibody (dilution 1:500; cat. no. SA00004-2; ProteinTech Group, Inc.) at room temperature for $1 \mathrm{~h}$. Freshly prepared 3,3'-diaminobenzidine (DAB) from a DAB Substrate kit (Abcam) was added for color development. ICH scoring was performed as previously described (18). Staining intensity was graded on a 0-3 scale as follows: 0 , absence of staining; 1 , weak staining; 2 , moderate staining; 3 , strong staining. The percentage of positive tumor cells was scored as follows: 0 , absence of tumor cells; $1,<33 \%$ positive tumor cells; $2,33-66 \%$ positive tumor cells; $3,>66 \%$ tumor cells. The IHC score (0-9) was calculated by multiplying the staining intensity by the percentage scores.

Cell culture. L02 cells were purchased from Xiangya Central Experiment Laboratory (Changsha, China). Li-7, Huh-7, SNU-387 and Hep3B cells were obtained from the Cell Bank of the Chinese Academy of Sciences (Shanghai, China). Human umbilical vein endothelial cells (HUVECs) were purchased from the China Center for Typical Culture Collection. L02, SNU-387 and Li-7 cells were cultured in RPMI-1640 medium (Gibco; Thermo Fisher Scientific, Inc.) containing 10\% fetal bovine serum (FBS; Gibco; Thermo Fisher Scientific, Inc.). Huh-7 cells were cultured in DMEM (Invitrogen; Thermo Fisher Scientific, Inc.) containing 10\% FBS. Hep3B cells were cultured in Minimum Essential medium (Gibco; Thermo Fisher Scientific, Inc.) containing 10\% FBS.

siRNA, plasmids, cell grouping and transfection. The full-length clone DNA of human DEPDC1 (NM_001114120.3; 2,436 bp) was obtained from FulenGen and subsequently cloned into the pcDNA3.1 plasmid vector by SunBio (Shanghai, China). DEPDC1 siRNA, CCL20 siRNA, CCR6 siRNA and the scrambled negative control (NC) used in this study were designed and synthesized by Shanghai GenePharma Co., Ltd. DEPDC1 short hairpin RNA (DEPDC1 shRNA) and scrambled negative shRNA (shNC) were designed, synthesized and cloned into the pMAGic1.1 plasmid vector by SunBio (Shanghai, China). The sequences of siRNA and shRNA are listed in Table I.

To determine the effects of DEPDC1 downregulation, Huh-7 and SNU-387 cells were divided to two groups: A scrambled NC group and a DEPDC1 siRNA group. Huh-7 and SNU-387 cells $\left(3 \times 10^{5}\right.$ cells $\left./ 2 \mathrm{ml}\right)$ were seeded into 6 -well plates. When cells reached $70-90 \%$ confluence, they were transfected. A total of 30 pmol NC or DEPDC1 siRNA with $7.5 \mu$ l Lipofectamine ${ }^{\circledR}$ RNAiMAX reagent (Invitrogen; Thermo Fisher Scientific, Inc.) in $250 \mu \mathrm{l}$ Opti-MEM ${ }^{\circledR}$ medium was added into each well. After $6 \mathrm{~h}$, the transfection medium was replaced with DMEM or RPMI-1640 medium. Further analyses were performed after incubation for $72 \mathrm{~h}$.

To generate a stable DEPDC1-suppressed Huh-7 cell line, Huh-7 cells were transfected with the shNC and DEPDC1 shRNA plasmid vectors. Huh-7 cells $\left(3 \times 10^{5}\right.$ cells $\left./ 2 \mathrm{ml}\right)$ were seeded into 6 -well plates. When cells reached 70-90\% confluence, $2.5 \mu \mathrm{g}$ shNC or DEPDC1 shRNA with $8 \mu$ l Lipofectamine ${ }^{\circledR} 2000$ reagent (Invitrogen; Thermo Fisher

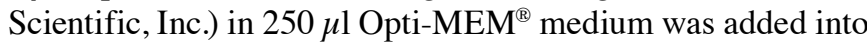
each well. After $8 \mathrm{~h}$, the transfection medium was replaced with DMEM. After $72 \mathrm{~h}$, the cells were selected using $6 \mu \mathrm{g} / \mathrm{ml}$ puromycin for 4 weeks. 
Table I. The sequences of siRNA, shRNA and RT-qPCR primers.

\begin{tabular}{|c|c|}
\hline Name & Sequences \\
\hline DEPDC1 siRNA sense & 5'-UCAGAACAAUCGCAGAUUAUUTT-3' \\
\hline DEPDC1 siRNA antisense & 5'-AAUAAUCUGCGAUUGUUCUGATT-3' \\
\hline CCR6 siRNA sense & 5'-GACCAGUGAGACCGCAGAUAATT-3' \\
\hline CCR6 siRNA antisense & 5'-UUAUCUGCGGUCUCACUGGUCTT-3' \\
\hline NC sense & 5'-UUCUCCG AACGUGUCACGUTT-3' \\
\hline $\mathrm{NC}$ antisense & 5'-ACGUGACACGUUCGGAGAATT-3' \\
\hline DEPDC1 shRNA sense & $\begin{array}{l}\text { 5'-CCGGTCAGAACAATCGCAGATTATTCTCGAGAATAATCTGCGATTGTTCTGATT } \\
\text { TTTG-3' }\end{array}$ \\
\hline DEPDC1 shRNA antisense & $\begin{array}{l}\text { 5'-AATTCAAAAAACCGGTCAGAACAATCGCAGATTATTCTCGAGAATAATCTGCG } \\
\text { ATTGTTCTGA-3' }\end{array}$ \\
\hline shNC sense & $\begin{array}{l}\text { 5'-CCGGTTCTCCGAACGTGTCACGTTTCAAGAGAACGTGACACGTTCGGAGAAT } \\
\text { TTTTG-3' }\end{array}$ \\
\hline shNC antisense & $\begin{array}{l}\text { 5'-AATTCAAAAATTCTCCGAACGTGTCACGTTCTCTTGAAACGTGACACGTTCGG } \\
\text { AGAA-3' }\end{array}$ \\
\hline DEPDC1 forward & 5'-CCGAACATAGAAGGACAA -3' \\
\hline DEPDC1 reverse & 5'-CTCTTGGTCTTGAACAGT-3' \\
\hline$\beta$-actin forward & 5'-CATGTACGTTGCTATCCAGGC-3' \\
\hline$\beta$-actin reverse & 5'-CTCCTTAATGTCACGCACGAT-3' \\
\hline CCL20 forward & 5'-CTCCTGGCTGCTTTGATGTC-3' \\
\hline CCL20 reverse & 5'-ATTTGCGCACACAGACAACT-3' \\
\hline CCR6 forward & 5'-CCTGGGGAATATTCTGGTGGTGA-3' \\
\hline CCR6 reverse & 5'-CATCGCTGCCTTGGGTGTTGTAT-3' \\
\hline
\end{tabular}

DEPDC1, DEP domain containing 1; siRNA, small interfering RNA; CCR6, chemokine (C-C motif) receptor 6; NC, negative control; shRNA, short hairpin RNA; shNC, short hairpin NC; CCL20, chemokine (C-C motif) ligand 20.

To assess the effect of DEPDC1 upregulation, Li-7 and Hep3B cells were divided into two groups: A pcDNA3.1 control group (pcDNA3.1) and a pcDNA3.1 DEPDC1 expression vector group (DEPDC1). Li-7 and Hep3B cells $\left(3 \times 10^{5}\right.$ cells $\left./ 2 \mathrm{ml}\right)$ were seeded into 6 -well plates. When the cells reached $70-90 \%$ confluence, $2.5 \mu \mathrm{g}$ pcDNA3.1 or DEPDC1 plasmids with $8 \mu$ l Lipofectamine ${ }^{\circledR} 2000$ reagent in $250 \mu \mathrm{l}$ Opti-MEM ${ }^{\circledR}$ medium were added into each well. After $8 \mathrm{~h}$, the transfection medium was replaced with RPMI-1640 medium or MEM. Further analyses were performed after incubation for $72 \mathrm{~h}$.

For rescue experiments, Li-7 and Hep3B cells were divided into four groups: Cells co-transfected with pcDNA3.1 control vectors and the scrambled negative control (pcDNA3.1+NC group); cells co-transfected with DEPDC1 expression vectors and the scrambled negative control (DEPDC1+NC group); cells co-transfected with DEPDC1 expression vectors and CCL20 siRNA (DEPDC1+CCL20 siRNA group); and cells co-transfected with DEPDC1 expression vectors and CCR6 siRNA (DEPDC1+CCR6 siRNA group). Li-7 and Hep3B cells $\left(3 \times 10^{5}\right.$ cells $\left./ 2 \mathrm{ml}\right)$ were seeded into 6 -well plates. When cells reached $70-90 \%$ confluence, 75 pmol NC, CCL20 siRNA or CCR6 siRNA in addition to $2.5 \mu \mathrm{g}$ pcDNA3.1 control vectors or DEPDC1 expression vectors in $250 \mu \mathrm{l} \mathrm{Opti-MEM}{ }^{\circledR}$ medium were added into each well. After $8 \mathrm{~h}$, the transfection medium was replaced with RPMI-1640 medium or MEM. Further analyses were performed after incubation for $72 \mathrm{~h}$.

Western blotting. After treatment, cells were collected and lysed with ice-cold RIPA lysis buffer (cat. no. AR0105; Wuhan Boster Biological Technology, Ltd.). The cell suspension was centrifuged at $12,000 \times \mathrm{g}$ for $15 \mathrm{~min}$, and the supernatant was aspirated and placed in a fresh tube. A bicinchoninic acid protein concentration assay kit (cat. no. AR0146; Wuhan Boster Biological Technology, Ltd.) was used to determine the protein concentration of each cell lysate. Total protein $(30 \mu \mathrm{g})$ from each cell lysate was adjusted to an equal volume using loading buffer and denatured at $100^{\circ} \mathrm{C}$ for 5 min. Each cell sample was separated via 10\% SDS-PAGE and subsequently electro-transferred to PVDF membranes (cat. no. AR0136-04; Wuhan Boster Biological Technology, Ltd.). The membranes were then blocked with $5 \%$ non-fat milk for $1 \mathrm{~h}$ at room temperature. After blocking, the membranes were incubated with the following primary antibodies overnight at $4^{\circ} \mathrm{C}$ : Rabbit anti-human DEPDC1 (dilution 1:500; cat. no. PA5-34864) and goat anti-human CCL20 (dilution 1:2,000; cat. no. PA5-47517; both from Invitrogen; Thermo Fisher Scientific, Inc.), rabbit anti-human CCR6 (dilution 1:1,000; cat. no. ab227036; Abcam), rabbit anti-human p-AKT (dilution 1:1,500; cat. no. P00024-6; Wuhan Boster Biological Technology, Ltd.), rabbit anti-human AKT (dilution 1:1,000; 
cat. no. 10176-2-AP), rabbit anti-human c-myc (dilution 1:2,000; cat. no. 10828-1-AP), rabbit anti-human cyclin E1 (dilution 1:1,000; cat. no. 11554-1-AP) and mouse anti-human $\beta$-actin (dilution 1:5,000; cat. no. 66009-1-Ig; all from ProteinTech Group, Inc.). After incubation, the membranes were washed three times with TBST and incubated with horseradish peroxidase (HRP)-conjugated AffiniPure goat anti-rabbit (dilution 1:5,000; cat. no. SA00001-2; ProteinTech Group, Inc.), HRP-conjugated AffiniPure rabbit anti-goat (dilution 1:5,000; cat. no. SA00001-4; ProteinTech Group, Inc.) and HRP-conjugated AffiniPure goat anti-mouse (dilution 1:5,000; cat. no. SA00001-1; ProteinTech Group, Inc.) at room temperature for $1 \mathrm{~h}$. After incubation, the membranes were washed three times with TBST. Protein bands were visualized using an enhanced chemiluminescence substrate (cat. no. 34095; Thermo Fisher Scientific, Inc.). Grey values of the protein bands were semi-quantified by ImageJ software (version 1.43; National Institutes of Health). Grey values were normalized to $\beta$-actin and expressed as relative densities.

$R T$ - $q P C R$. Total RNA was isolated from tissues and cultured cells using RNAiso Plus (cat. no. 9108; Takara Biotechnology Co., Ltd.) in accordance with the manufacturer's protocol. Sample integrity and purity was analyzed via agarose gel electrophoresis and the value of A260/A280. RT was performed using M-MLV-RTase (cat. no. M1705; Promega Corporation) at $42^{\circ} \mathrm{C}$ for $1 \mathrm{~h}$ with an $11-\mu \mathrm{l}$ system $(4 \mu \mathrm{l} 5 \mathrm{xRT}$ buffer, $2 \mu \mathrm{l}$ $10 \mathrm{mM}$ dNTPs, $0.5 \mu \mathrm{l}$ RNasin, $1 \mu \mathrm{l}$ M-MLV-RTase and $3.5 \mu \mathrm{l}$ DEPC- $\mathrm{H}_{2} \mathrm{O}$ ). The expression of target genes was analyzed using SYBR Master Mixture (cat. no. DRR041B; Takara Biotechnology Co., Ltd.). The thermocycling conditions were as follows: Pre-denaturation for $15 \mathrm{sec}$ at $95^{\circ} \mathrm{C}, 45$ cycles of denaturation for $5 \mathrm{sec}$ at $95^{\circ} \mathrm{C}$, annealing at $60^{\circ} \mathrm{C}$ for $30 \mathrm{sec}$ and extension at $60^{\circ} \mathrm{C}$ for $30 \mathrm{sec}$. The sequences of all primers used are presented in Table I. The relative expression of the target genes was normalized to $\beta$-actin using the $2^{-\Delta \Delta \mathrm{Cq}}$ analysis method (19).

Cell viability assay. After treatment, cells were inoculated in 96-well plates $\left(1 \times 10^{4}\right.$ cells/well) and further cultured for 1, 2, 3 or 4 days. Cell Counting Kit-8 solution (CCK-8; cat. no. C0038; Beyotime Institute of Biotechnology) was thawed in a water bath at $37^{\circ} \mathrm{C}$. A total of $10 \mu \mathrm{l} \mathrm{CCK-8} \mathrm{solu-}$ tion was then added to each well of the plate. Cells were subsequently incubated for $2 \mathrm{~h}$ at $37^{\circ} \mathrm{C}$. After incubation, absorbance was measured at $450 \mathrm{~nm}$ using a microplate reader (Beckman Coulter, Inc.).

$E d U$ assay. Cell proliferation was determined using the BeyoClick $^{\mathrm{TM}}$ EdU Cell Proliferation kit with Alexa Fluor 594 (cat. no. C0078S; Beyotime Institute of Biotechnology). Cells were then washed with PBS and fresh medium (RPMI-1640 medium, DMEM or MEM) containing $10 \mu \mathrm{M}$ EdU was added. Cells were subsequently incubated for $2 \mathrm{~h}$ at $37^{\circ} \mathrm{C}$ in $5 \% \mathrm{CO}_{2}$ and washed with PBS to remove the free EdU probe and medium. Cells were then fixed in $4 \%$ paraformaldehyde at room temperature for $15 \mathrm{~min}$ and stained with DAPI for $5 \mathrm{~min}$. After an additional wash in PBS, positive cells were observed under a Olympus IX71 fluorescent microscope (Olympus Corp., Tokyo, Japan; magnification, x200) and analyzed using
ImageJ software (version 1.43; National Institutes of Health). The mean numbers of EdU-positive cells were calculated from three images of each group (20).

Colony formation assay. After treatment, cells were seeded into 6-well plates (400 cells/well) and further cultured at $37^{\circ} \mathrm{C}$. After incubation for 14 days, the cells were washed with PBS, fixed with $4 \%$ paraformaldehyde at room temperature for $15 \mathrm{~min}$, and stained with $0.2 \%$ crystal violet solution at room temperature for $20 \mathrm{~min}$. The number of colonies was counted with an inverted fluorescence microscope (Nikon TE2000; Nikon Corp.).

HCC cell invasion assay. HCC cell invasion activities were determined using a 24-well Transwell chamber (cat. no. 3577; Corning Costar Corp.) with Matrigel (cat. no. 356234; BD Biosciences). After treatment, cells were seeded into the upper chamber of the Transwell equipment at a density of $1 \times 10^{5}$ cells per insert. A total of $500 \mu 1$ of RPMI-1640, DMEM or MEM with $10 \%$ FBS was added to the lower chamber of the Transwell insert. After incubation for $48 \mathrm{~h}$, cells remaining on the upper chamber of the Transwell insert were removed. Cells on the underside of the chamber were fixed with $4 \%$ paraformaldehyde and stained with $0.1 \%$ crystal violet. Invaded cells were subsequently counted under a microscope (magnification, x200) in six different fields of view for each sample.

In vitro HUVEC tube formation assay. Li-7 and Hep3B cells transfected with pcDNA3.1 or DEPDC1 expression vectors, or co-transfected with NC or CCL20 siRNA were further incubated for $72 \mathrm{~h}$. Tumor cell-conditioned medium (TCM) was prepared as previously described $(21,22)$. The medium was removed, and the cells were washed in triplicate with PBS. Serum-free RPMI-1640 medium or MEM was then added to each well of the plate. After incubation for $24 \mathrm{~h}$, TCM was collected and stored at $-80^{\circ} \mathrm{C}$. Prior to the tube formation assay, each well of a 96 -well plate was pre-coated with Matrigel (cat. no. 3432-005-01; R\&D Systems, Inc.). HUVECs or HUVECs pretreated with NC or CCR6 siRNA $\left(2 \times 10^{4}\right.$ cells $\left./ 100 \mu \mathrm{l}\right)$ were then added to each well of the plate. After incubation for $4 \mathrm{~h}$, the branch points of the formed tubes were scanned under a inverted fluorescence microscope (Nikon TE2000; Nikon Corp.) and quantitated in at least 5 microscopic fields.

HUVEC invasion assays. HUVECs invasion activities were determined using a 24-well Transwell chamber (cat. no. 3577; Corning Costar Corp.) with Matrigel (cat. no. 356234; BD Biosciences). Cells $\left(1 \times 10^{5}\right)$ prepared in $400 \mu 1$ serum-free media were seeded into the upper chamber of the Transwell insert. A total of $600 \mu \mathrm{l} \mathrm{TCM}$ was added to the lower chamber of the Transwell insert. After incubation for $48 \mathrm{~h}$, the cells remaining in the upper chamber of the Transwell insert were removed. Cells on the underside of the chamber were fixed with $4 \%$ paraformaldehyde at room temperature for $30 \mathrm{~min}$ and stained with $0.1 \%$ crystal violet at room temperature for $20 \mathrm{~min}$. Invaded cells were subsequently counted under a inverted fluorescence microscope (Nikon TE2000; Nikon Corp.) (magnification, x200) in six different fields of view for each sample. 
Nude mice tumour xenograft model. BALB/c nude mice $(\mathrm{n}=5)$ at 4-5 weeks of age (weighing 20 g) were obtained from Vital River Laboratories Co., Ltd., and randomly divided into two groups. All mice were maintained under specific pathogen-free conditions (temperature $25 \pm 10^{\circ} \mathrm{C}$ and relative humidity $60 \pm 10 \%$ ) in laminar flow cabinets. All mice were housed with a 12-h light/dark cycle and autoclaved food/water was provided freely. The stable DEPDC1-suppressed Huh-7 cell line (DEPDC1 shRNA) and the shRNA control Huh-7 cell line (shNC; $2 \times 10^{6}$ cells $/ 100 \mu \mathrm{l}$ PBS) were inoculated subcutaneously in the left dorsal flank and the right flank of BALB/c nude mice, respectively. The width and length of the tumors were measured weekly and the volume was calculated using the following equation: (length $\mathrm{x}$ width ${ }^{2}$ )/2. After five weeks of injections, all mice were euthanized by $\mathrm{CO}_{2}$ asphyxiation and the tumors were excised.

Microarray analysis. Huh-7 cells were transfected with DEPDC1 siRNA or NC siRNA and further cultured for $72 \mathrm{~h}$. After incubation, total RNA was isolated using the TRIzol Reagent (cat. no. 15596026; Invitrogen; Thermo Fisher Scientific, Inc.). The integrity and purity of RNA was analyzed via agarose gel electrophoresis and the value of A260/A280. cDNA was subsequently synthesized from total RNA and hybridized to the Affymetrix Gene Chip Human Gene 1.0 ST Array (cat. no. 901085; Affymetrix; Thermo Fisher Scientific, Inc.). Differentially expressed genes were analyzed using the Expression Console and Transcriptome Analysis Console v3.0 (Affymetrix; Thermo Fisher Scientific, Inc.). DAVID Bioinformatics Resources 6.7 (https://david.ncifcrf. gov/) was used to perform GO annotation and KEGG pathway analyses. Raw data has been deposited in the GEO database (GEO accession no. GSE122124).

Statistical analysis. All data were analyzed using IBM SPSS 21.0 software (IBM Corp.) and all experiments were repeated at least three times. Data were presented as the mean \pm standard deviation. Significant differences between groups were determined using a Student's t-test or one-way analysis of variance (ANOVA) and Dunnett's multiple comparison post hoc test. The correlation between DEPDC1 and CCL20 or CCR6 expression in HCC tissue was determined via Pearson's correlation. $\mathrm{P}<0.05$ was considered to indicate a statistically significant difference.

\section{Results}

DEPDC1 knockdown suppresses HCC tumourigenesis in vitro and in vivo. To assess the molecular role of DEPDC1 in HCC, the expression of DEPDC1 was analyzed in four human HCC cell lines (Li-7, Huh-7, SNU-387 and Hep3B) and one normal human hepatic cell line (L02) via western blotting. The results revealed that DEPDC1 protein was primarily detected in $\mathrm{HCC}$ cell lines and a decreased expression was detected in L02 cells (Fig. 1A). Huh-7 and SNU-387 cells were transfected with a scrambled NC and DEPDC1 siRNA, the expression of DEPDC1 was determined by RT-qPCR and western blotting. As presented in Fig. 1B, DEPDC1 siRNA significantly suppressed DEPDC1 mRNA and protein expression. The results of the CCK- 8 assay demonstrated that DEPDC1 knockdown significantly inhibited Huh-7 and SNU-387 cell viability (Fig. 1C). Following the EdU assay, a decreased number of EdU-positive cells were observed in Huh-7 and SNU-387 cells transfected with DEPDC1 siRNA compared with those transfected with the NC (Fig. 1D). The results of the colony formation and cell invasion assays revealed that DEPDC1 knockdown significantly inhibited colony formation and cell invasion in Huh-7 and SNU-387 cells (Fig. 1E and F). The present study generated a stable DEPDC1-suppressed Huh-7 cell line using DEPDC1 shRNA vectors and puromycin. The results also revealed that the tumor volume of the DEPDC1 shRNA group was lower than that of the shNC group (Fig. 1G).

DEPDCl upregulation promotes HCC cell progression. To further confirm the molecular role of DEPDC1 in HCC, Li-7 and Hep3B cells were transfected with pcDNA3.1 and the pcDNA3.1 DEPDC1 expression vector. As presented in Fig. 2A, treatment with the DEPDC1 expression plasmid significantly upregulated DEPDC1 protein expression. The results of the CCK-8 assay demonstrated that DEPDC1 upregulation significantly induced Li-7 and Hep3B cell viability (Fig. 2B). Following the EdU assay, an increased number of EdU-positive cells were observed in Li-7 and Hep3B cells transfected with the pcDNA3.1 DEPDC1 expression vector compared with those transfected with the pcDNA3.1 control (Fig. 2C). The results of the colony formation and cell invasion assays revealed that DEPDC1 upregulation significantly increased colony formation and cell invasion in $\mathrm{Li}-7$ and Hep3B cells (Fig. 2D and E).

DEPDC1 downregulation affects gene expression at a global level. To assess the possible mechanisms of DEPDC1 in HCC progression, microarray analysis was performed using RNA isolated from NC or DEPDC1 siRNA-transfected Huh-7 cells. As presented in Fig. 3A and B, a total of 129 genes were downregulated (fold change, <-2-fold) and 252 genes were upregulated (fold change, $>2$-fold). In addition, GO annotation and KEGG pathway analysis were performed using the DAVID Bioinformatics Resource 6.7 (https://david.ncifcrf. gov/). The results of GO analysis revealed that DEPDC1 was involved in C-X-C chemokine binding (Fig. 3C), vasculature development (Fig. 3D), blood vessel development (Fig. 3D) and extracellular matrix (Fig. 3E). KEGG pathway analysis determined that downregulated and upregulated genes significantly enriched in cytokine-cytokine receptor interaction (Fig. 3F). According to the functional results of DEPDC1, GO analysis and KEGG pathway analysis, the CCL20/CCR6 axis was selected for further study. In recent years, the CCL20/CCR6 axis has been implicated in the growth and invasion of HCC cells and in HCV-associated HCC hepatic angiogenesis (14-16). Thus, the CCL20/CCR6 axis may serve a vital role in the DEPDC1-mediated progression of HCC. RT-qPCR was performed to validate the expression of CCL20 and CCR6. In congruence with microarray results, the expression of CCL20 and CCR6 in Huh-7 cells treated with DEPDC1 siRNA was significantly lower compared with the NC group (Fig. 3G). In addition, DEPDC1 knockdown significantly inhibited the protein expression of CCL20 and CCR6 (Fig. 3H). 

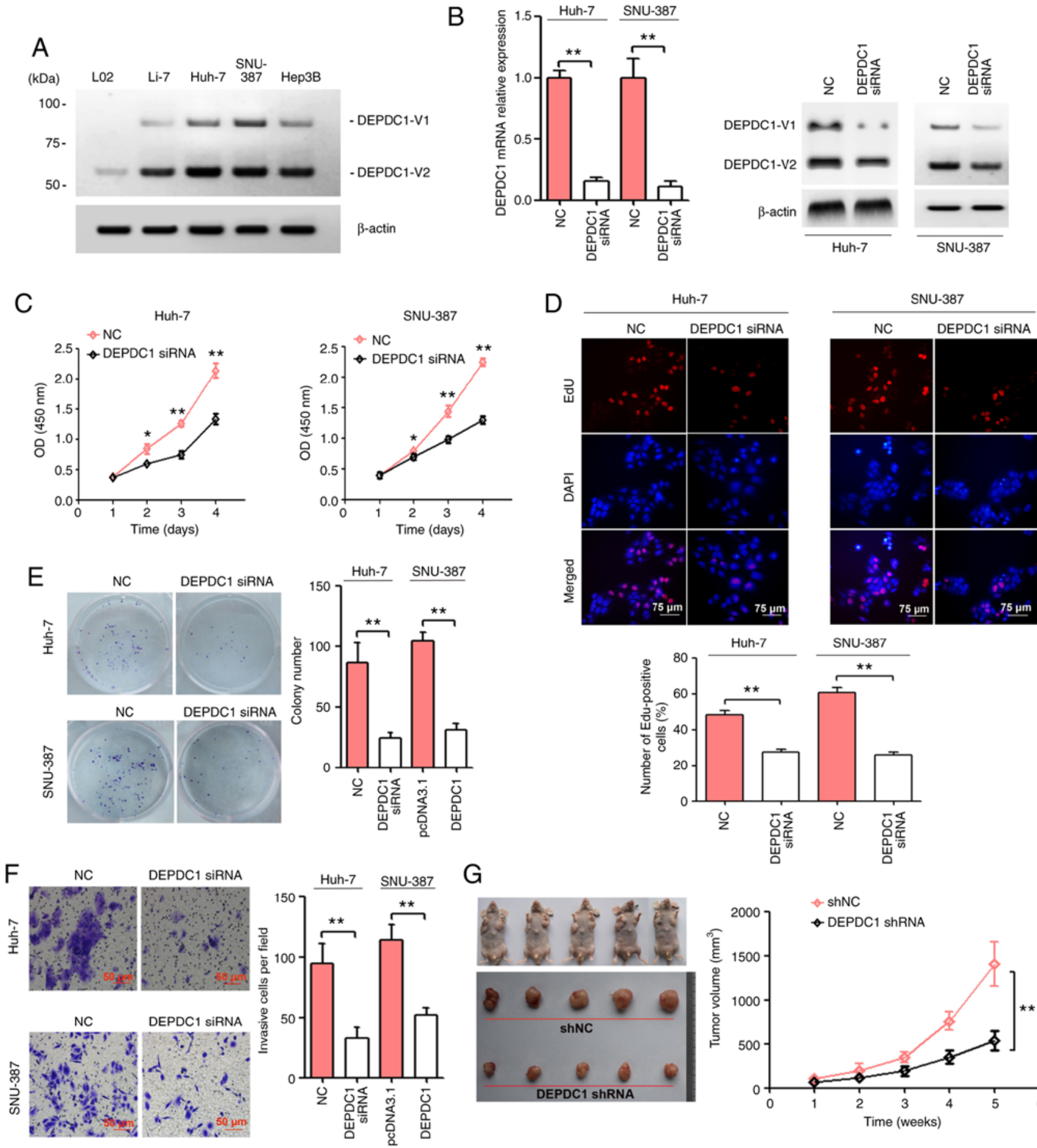

Figure 1. DEPDC1 knockdown suppresses HCC tumourigenesis in vitro and in vivo. (A) The protein expression of DEPDC1 in a normal human hepatic cell line (L02) and four human HCC cell lines (Li-7, Huh-7, SNU-387 and Hep3B) was detected by western blotting. (B) Huh-7 and SNU-387 cells were transfected with a scrambled negative control and DEPDC1 siRNA. After transfection for $72 \mathrm{~h}$, DEPDC1 mRNA and protein expression was confirmed via RT-qPCR and western blotting, respectively. Huh-7 and SNU-387 cells were transfected as described above. Cell growth was evaluated via (C) Cell Counting Kit-8, (D) EdU and (E) colony formation assays. Scale bar, $75 \mu \mathrm{m}$. (F) Cell invasion was determined by performing a Transwell assay. Scale bar, $50 \mu \mathrm{m}$. (G) Huh-7 cells transfected with DEPDC1 shRNA exhibited a significantly reduced tumor volume (5 per group). ${ }^{*} \mathrm{P}<0.05,{ }^{* *} \mathrm{P}<0.01$. DEPDC1, DEP domain containing 1; HCC, hepatocellular carcinoma; NC, negative control; siRNA, small interfering RNA; shRNA, short hairpin RNA; RT-qPCR, reverse transcription-quantitative PCR.

CCL2O and CCR6 expression is significantly increased in HCC tissue and cell lines. To verify the expression of DEPDC1, CCL20 and CCR6 mRNA in HCC tissue, RT-qPCR analysis was performed. The results revealed that DEPDC1, CCL20 and CCR6 mRNA was significantly upregulated in HCC tissue compared with matched adjacent normal liver tissue (Fig. 4A). In addition, CCL20 and CCR6 mRNA expression were positively correlated with that of DEPDC1 (Fig. 4B). Furthermore, IHC revealed that DEPDC1, CCL20 and CCR6 protein expression was significantly upregulated in HCC tissue compared with matched adjacent normal liver tissue (Fig. 4C and D). CCL20 and CCR6 protein expression were 
A
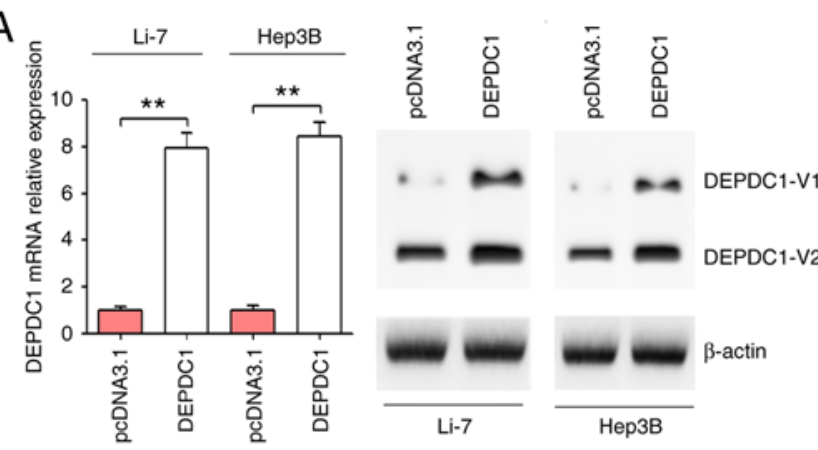

B
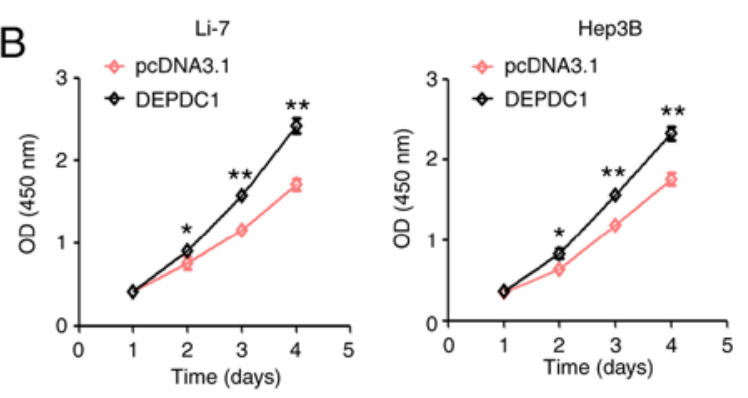

C
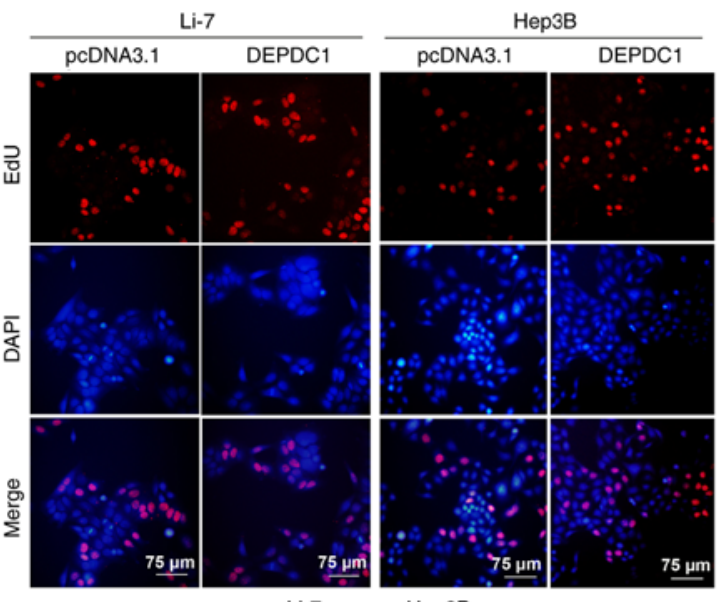

D

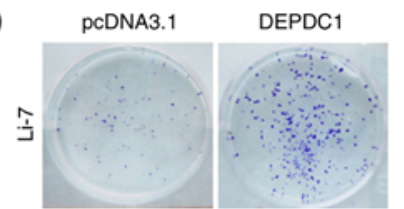

E

pcDNA3.1 DEPDC1

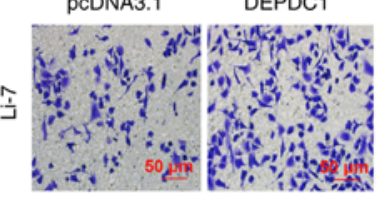

pcDNA3.

DEPDC1
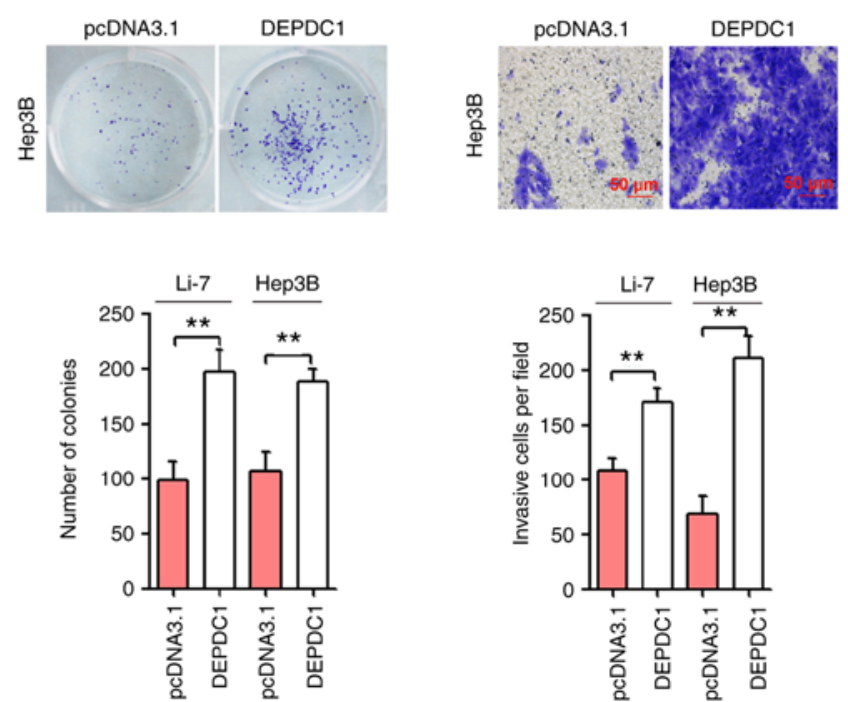

Figure 2. DEPDC1 upregulation promotes HCC cell progression. (A) Li-7 and Hep3B cells were transfected with pcDNA3.1 control and pcDNA3.1 DEPDC1 expression vectors. After transfection for $72 \mathrm{~h}, \mathrm{DEPDC} 1 \mathrm{mRNA}$ and protein expression was confirmed via reverse RT-qPCR and western blotting, respectively. Li-7 and Hep3B cells were transfected as described above. Cell growth was evaluated via (B) Cell Counting Kit-8, (C) EdU and (D) colony formation assays . Scale bar, $75 \mu \mathrm{m}$. (E) Cell invasion was determined by performing a Transwell assay. Scale bar, $50 \mu \mathrm{m}$. ${ }^{*} \mathrm{P}<0.05,{ }^{* *} \mathrm{P}<0.01$. DEPDC1, DEP domain containing 1 ; HCC, hepatocellular carcinoma; RT-qPCR, reverse transcription-quantitative PCR.

also positively correlated with DEPDC1 protein expression (Fig. 4E). To determine whether CCL20 and CCR6 protein was also upregulated in HCC cell lines, western blotting was performed. As presented in Fig. 4F, CCL20 and CCR6 protein expression were significantly upregulated in four human HCC cell lines (Li-7, Huh-7, SNU-387 and Hep3B) when compared with $\mathrm{L} 02$ cells.

CCL20 or CCR6 knockdown by siRNA reverses the effect of DEPDC1 overexpression in HCC cells. To confirm the effect of DEPDC1 upregulation on CCL20 and CCR6, the protein expression of CCL20 and CCR6 in Li-7 and Hep3B cells transfected with pcDNA3.1 and DEPDC1 expression vectors were confirmed via western blotting. As presented in Fig. S1, DEPDC1 upregulation in Li-7 and Hep3B cells significantly induced CCL20 and CCR6 protein expression. Furthermore, CCL20 knockdown via siRNA reversed the effect of DEPDC1 overexpression on CCL20 and CCR6 expression in Li-7 and Hep3B cells (Fig. S2). Additionally, CCR6 knockdown via
siRNA reversed the effect of DEPDC1 overexpression on the expression of CCR6 in Li-7 and Hep3B cells (Fig. S2). To ascertain whether DEPDC1 regulated HCC progression by mediating the CCL20/CCR6 axis, CCL20 and CCR6 were inhibited using siRNA in Li-7 and Hep3B cells transfected with DEPDC1 expression vectors. As presented in Fig. 5A, CCL20 or CCR6 knockdown reversed the increase in cell viability caused by DEPDC1 upregulation. In addition, the results revealed that CCL20 or CCR6 knockdown reversed the increase in the number of EdU-positive cells caused by DEPDC1 upregulation (Fig. 5B). As presented in Fig. 5C and D, CCL20 or CCR6 knockdown reversed the DEPDC1-mediated increase of colony formation and invasion. In normal breast cells, the CCL20/CCR6 axis may induce the expression of cell cycle-associated proteins (c-myc, c-Fos and cyclin E1) by inducing the activation of the PI3K/Akt pathway (23). The results of the present study revealed that DEPDC1 upregulation induced the expression of p-Akt, c-Myc and cyclin E1, while CCL20 or CCR6 knockdown reversed this effect (Fig. 5E). 
A

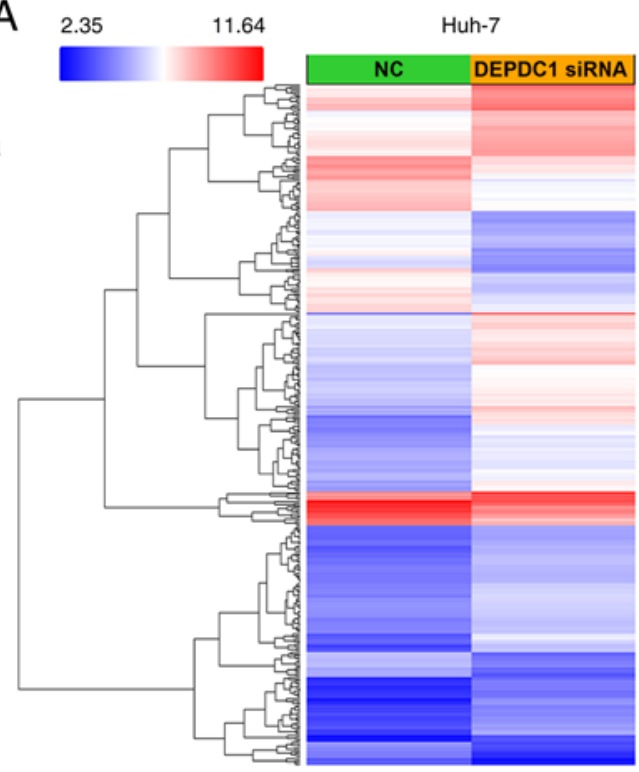

D
$\mathrm{B} \backsim$ Upregulated genes

- Downregulated genes

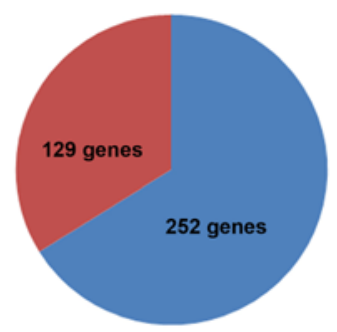

C

\begin{tabular}{|c|c|c|}
\hline & \multicolumn{2}{|c|}{$-\operatorname{Lg} p$} \\
\hline GO: Molecular function & 2 & \\
\hline Glycosaminoglycan binding & & \\
\hline Heparin binding & & \\
\hline Pattern binding & & \\
\hline $\begin{array}{l}\text { Polysaccharide binding } \\
\text { Carbohydrate binding }\end{array}$ & & \\
\hline
\end{tabular}

Extracellular ming Cadmium ion binding Endopeptidase inhibitor activity Peptidase inhibitor activity Enzyme inhibitor activity Calcium ion binding

Ligase activity, forming carbon-sulfur bonds Copper ion binding Growth factor binding Acid-thiol ligase activity Cytokine binding Butyrate-CoA ligase activity C-X-C chemokine binding PDZ domain binding Chemokine binding

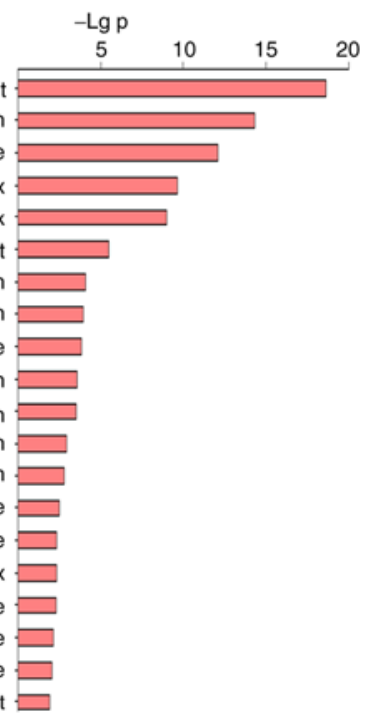

F

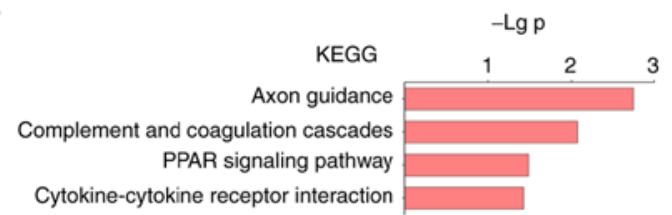

G

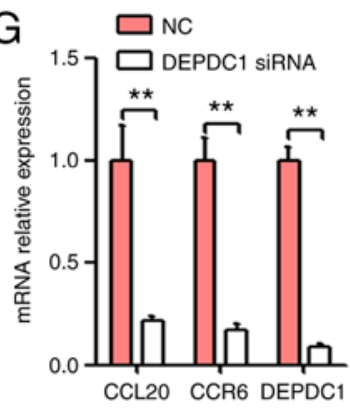

GO: Cellular component

Extracellular region part Extracellular region Extracellular space Extracellular matrix Proteinaceous extracellular matrix Extracellular matrix part Cell fraction Insoluble fraction Basement membrane Vesicle lumen

Membrane fraction Platelet alpha granule lumen Cytoplasmic membrane-bounded vesicle lumen Secretory granule Plasma lipoprotein particle Protein-lipid complex Platelet alpha granule Cytoplasmic vesicle $=$ Cytoplasmic vesicle part $=$

$\mathrm{H}$

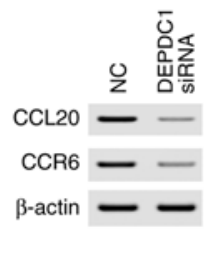

Figure 3. Microarray analysis of Huh-7 cells transfected with NC and DEPDC1 siRNA. (A) Heat map of differentially expressed genes with a-fold change of $>2$ or $<-2$ in DEPDC1 siRNA relative to the NC. (B) A total of 252 genes were upregulated with-fold changes $>2$, and 129 genes were downregulated with-fold changes <-2. GO (C) molecular function, (D) biological process and (E) cellular component term analyses of up- and downregulated genes in DEPDC1 siRNA relative to the NC. (F) KEGG analysis of up- and downregulated genes in DEPDC1 siRNA relative to the NC. (G) RT-qPCR and (H) western blotting analyses of selected candidate genes in Huh-7 cells transfected with DEPDC1 siRNA and NC. ${ }^{* *} \mathrm{P}<0.01$. NC, negative control; DEPDC1, DEP domain containing 1; siRNA, small interfering RNA; GO, Gene Ontology; KEGG, Kyoto Encyclopedia of Genes and Genomes; RT-qPCR, reverse transcription-quantitative PCR.

DEPDC1 promotes HUVEC invasion and tube formation by regulating CCL2O in vitro. A capillary tube formation assay was performed in the present study to determine the biological significance of DEPDC1 in tumor angiogenesis. As presented in Fig. 6A and B, HUVECs incubated with
TCM from DEPDC1-transfected Li-7 and Hep3B cells developed more capillary-like structures and displayed an increased invasive tendency compared with those cultured in pcDNA3.1-transfected TCM. To confirm whether DEPDC1 regulates HUVEC invasion and tube formation 

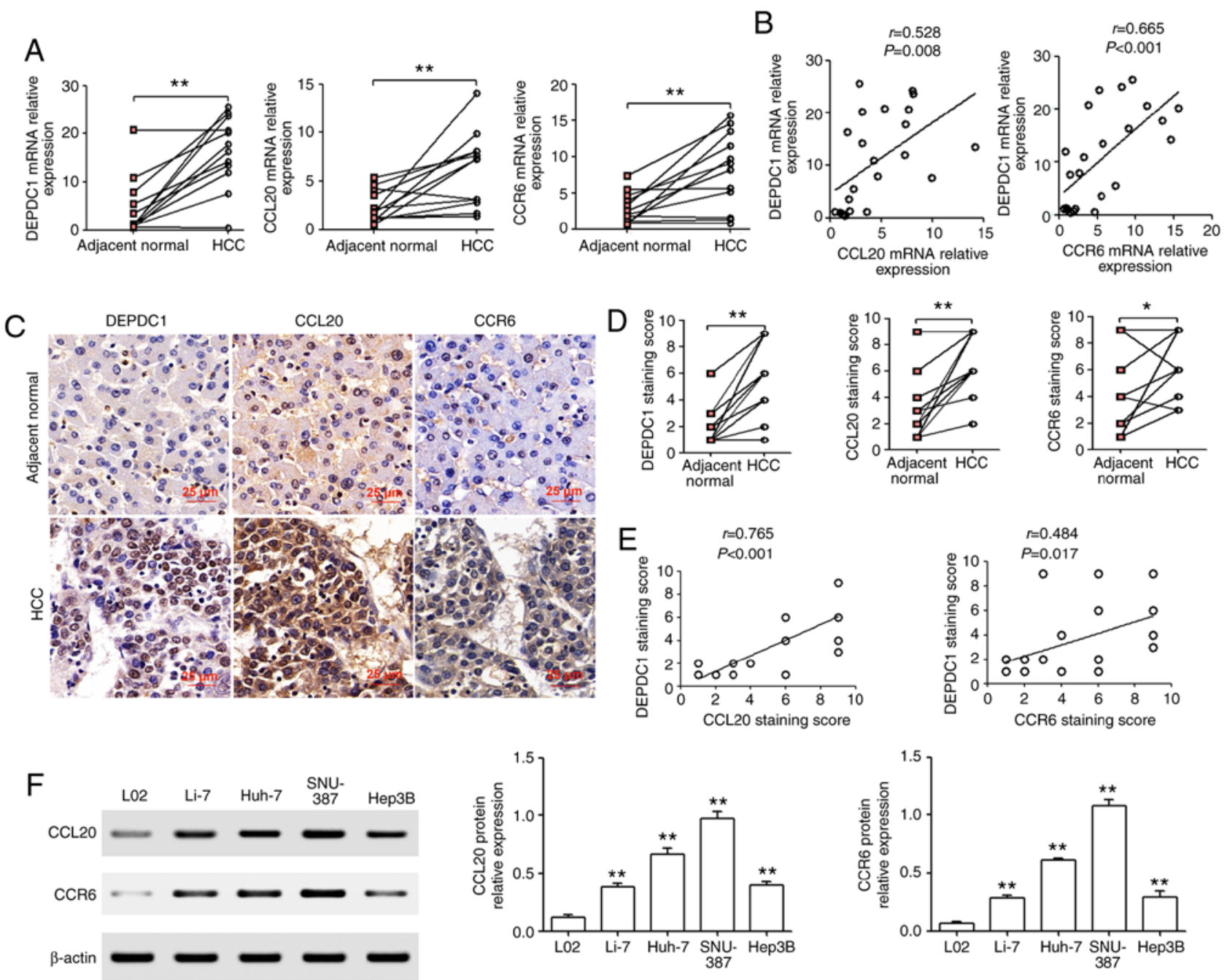

Figure 4. CCL20 and CCR6 are significantly increased in HCC tissues and cell lines. (A) RT-qPCR analysis of DEPDC1, CCL20 and CCR6 mRNA in HCC tissue and matched adjacent normal liver tissue. (B) Analysis of RT-qPCR results with significant Pearson correlation analysis of DEPDC1 with CCL20 and DEPDC1 with CCR6 in HCC. (C and D) Immunohistochemistry assays were used to determine the protein expression of DEPDC1, CCL20 and CCR6. Scale bar, $25 \mu \mathrm{m}$. (E) The correlation of DEPDC1 with CCL20 and CCR6 was determined via Pearson's correlation analysis. (F) Western blotting was performed to determine the protein expression of CCL20 and CCR6 in four human HCC cell lines (Li-7, Huh-7, SNU-387 and Hep3B) and one normal human hepatic cell line (L02). "P<0.05, ${ }^{* *} \mathrm{P}<0.01$. CCL20, chemokine (C-C motif) ligand 20; CCR6, chemokine (C-C motif) receptor 6; HCC, hepatocellular carcinoma; RT-qPCR, reverse transcription-quantitative PCR; DEPDC1, DEP domain containing 1.

by mediating CCL20 activity, TCM was collected from Li-7 and Hep3B cells co-transfected with DEPDC1 expression vectors and CCL20 siRNA. The results demonstrated that CCL20 knockdown reversed the effect of DEPDC1 upregulation on HUVEC angiogenesis and invasion (Fig. 6A and B).

CCR6 knockdown in HUVECs reverses the effect of DEPDC1 overexpression on angiogenesis and invasion. It has been previously revealed that endothelial CCR6 serves an important role in CCL20-induced blood vessel formation and invasion induced in HCC (16). Therefore, the present study assessed whether CCR6 knockdown in HUVECs affected the function of DEPDC1. As presented in Fig. 7A and B, HUVECs incubated with TCM from DEPDC1-transfected Li-7 and Hep3B cells developed more capillary-like structures and displayed an increased invasive tendency compared with those cultured in pcDNA3.1-transfected TCM. However, CCR6 knockdown in HUVECs reversed these effects. The results therefore indicated that DEPDC1 may promote HCC progression by regulating the CCL20/CCR6 axis (Fig. 8).

\section{Discussion}

Previous studies have indicated that DEPDC1 is elevated in several types of cancer and is implicated in tumorigenesis (6-10). It has also been revealed that DEPDC1 is upregulated in HCC tissue and may therefore be an independent predictor of HCC (11). Furthermore, DEPDC1 was revealed to be involved in the miR-130a-induced apoptosis and inhibition of proliferation in HepG2 cells (12). Qu et al (13) revealed that DEPDC1 promoted HCC cell proliferation and neoplasm metastasis. The present study also determined that DEPDC1 was positively correlated with the K-RAS signaling pathway, certain cancer-associated pathways and the WNT/ $\beta$-catenin signaling pathway. However, the mechanism of DEPDC1 in the regulation of HCC progression remains largely unknown. Therefore, further studies are required to 

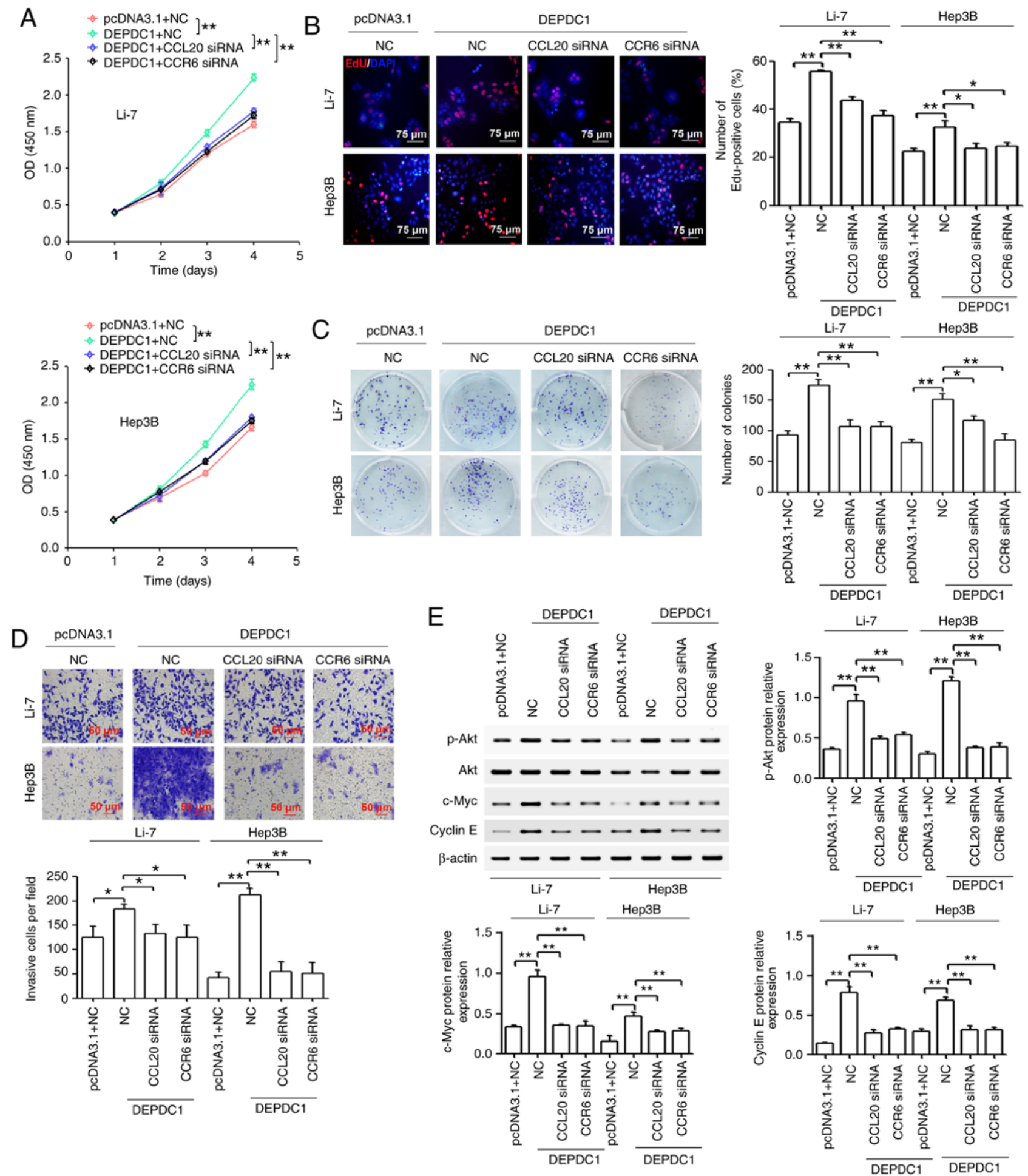

Figure 5. CCL20 or CCR6 knockdown partially reverses the effect of DEPDC1 overexpression on HCC cell proliferation, colony formation and invasion in vitro. Li-7 and Hep3B cells were treated with a combination of pcDNA3.1 and NC plasmids or a combination of DEPDC1 expression vectors with NC, CCL20 siRNA or CCR6 siRNA. (A) Cell Counting Kit-8 and (B) EdU assays were performed to determine cell proliferation. Scale bar, $75 \mu \mathrm{m}$. (C) Foci formation assays were utilized to observe colony formation. (D) Cell invasion was determined via a Transwell assay. Scale bar, $50 \mu \mathrm{m}$. (E) Western blotting was performed to detect the protein expression of p-Akt, Akt, c-myc and cyclin E. Each experiment was performed in triplicate. " $\mathrm{P}<0.05,{ }^{* *} \mathrm{P}<0.01$. CCL20, chemokine (C-C motif) ligand 20; CCR6, chemokine (C-C motif) receptor 6; DEPDC1, DEP domain containing 1; HCC, hepatocellular carcinoma; NC, negative control; siRNA, small interfering RNA; p-, phosphorylated.

assess the regulation and function of DEPDC1 to the benefit or patients with HCC.

The present study determined the expression of DEPDC1 in HCC cell lines. The results revealed that DEPDC1 was significantly upregulated in HCC cell lines. However, decreased expression of DEPDC1 was detected in the normal human hepatic cells (L02). Furthermore, DEPDC1 knockdown significantly suppressed proliferation, colony formation and invasion of Huh-7 and SNU-387 cells. In addition, DEPDC1 upregulation enhanced the proliferation, colony formation 


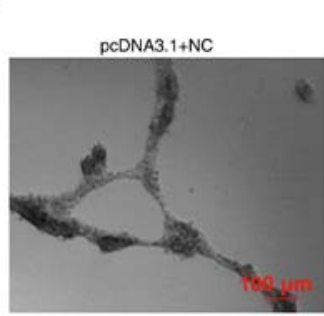

pCDNA3.1+NC

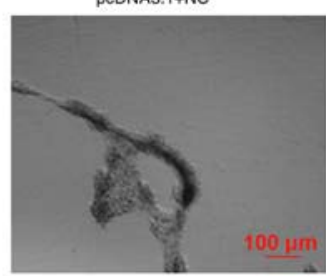

Li.7 conditioned media DEPDC $1+\mathrm{NC}$

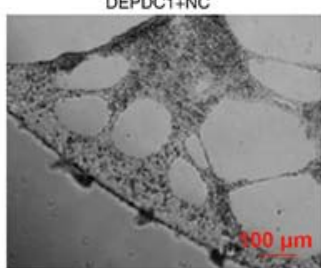

Hep38 conditioned media DEPDC $1+N C$

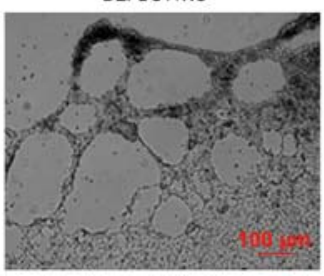

DEPDC1+CCL20 siRNA

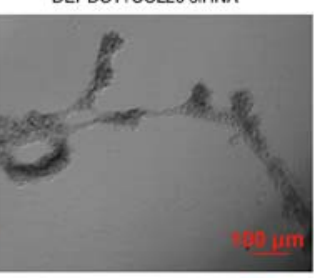

ep3B conditioned media

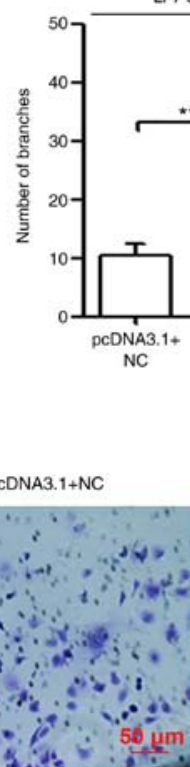

Li-7 conditioned media
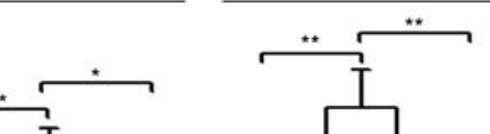

-

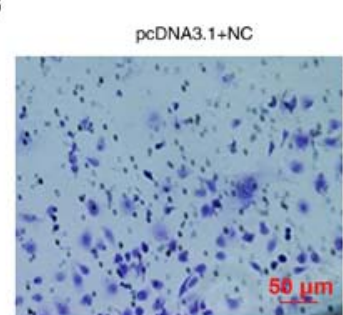

Li-7 conditioned media

DEPDC $1+N C$

DEPDC1+CCL20 SIRNA
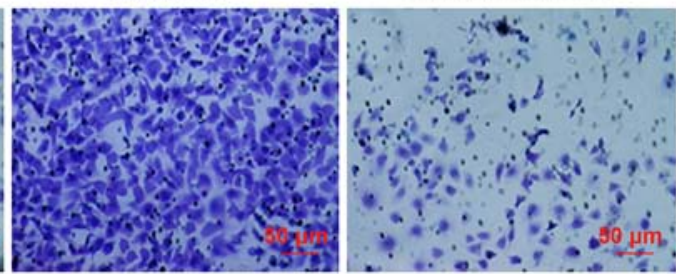

Hep3B conditioned media

pCDNA3.1+NC

DEPDC $1+N C$

DEPDC1+CCL20 SIRNA
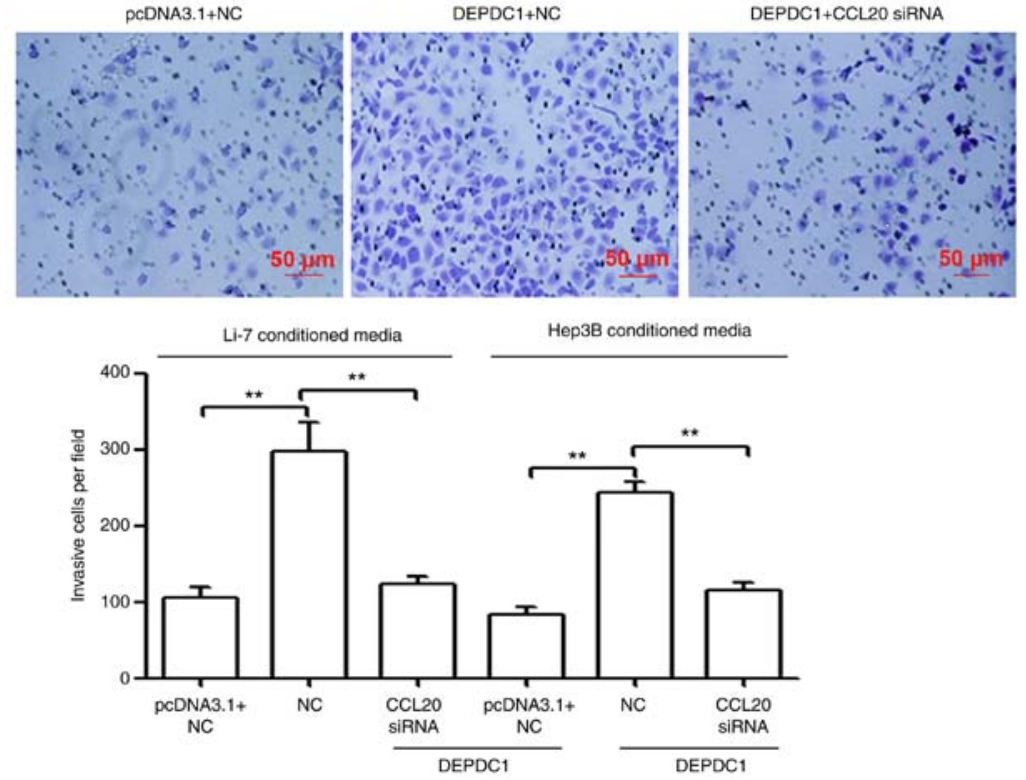

Figure 6. DEPDC1 upregulation induces tumor angiogenesis and invasion by regulating CCL20 in vitro. (A) TCM was collected from Li-7 and Hep3B cells treated with a combination of pcDNA3.1 and NC plasmids or a combination of DEPDC1 expression vectors and NC or CCL20 siRNA. A tube formation assay was performed using HUVECs with the indicated conditioned media. Scale bar, $100 \mu \mathrm{m}$. (B) The invasion of HUVECs was determined via a Transwell assay. Each experiment was performed in triplicate. Scale bar, $50 \mu \mathrm{m}$. ${ }^{*} \mathrm{P}<0.05,{ }^{* *} \mathrm{P}<0.01$. DEPDC1, DEP domain containing 1; CCL20, chemokine (C-C motif) ligand 20; TCM, tumor cell-conditioned medium; NC, negative control; siRNA, small interfering RNA; HUVECs, human umbilical vein endothelial cells. 
A

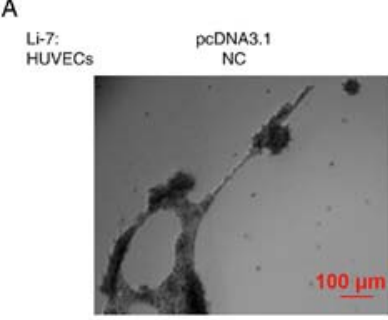

Hep3B:
HUVECS:

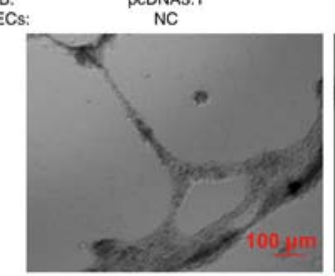

Li.7 conditioned media

DEPDC1
NC

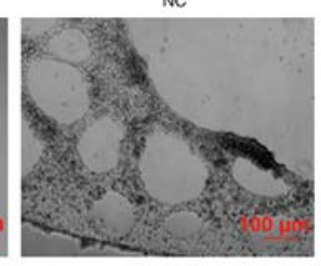

Hep3B conditioned media DEPDC
NC

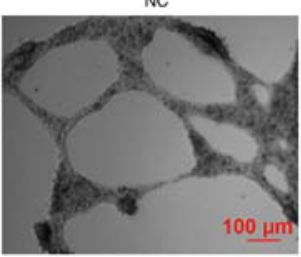

DEPDC1

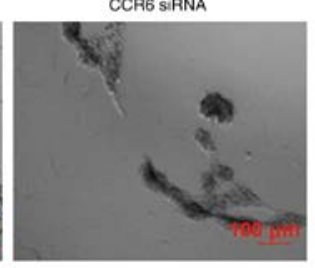

DEPDC1

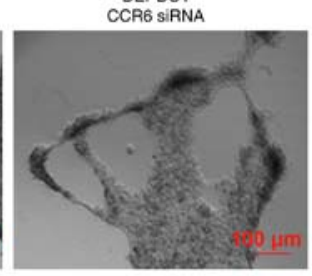

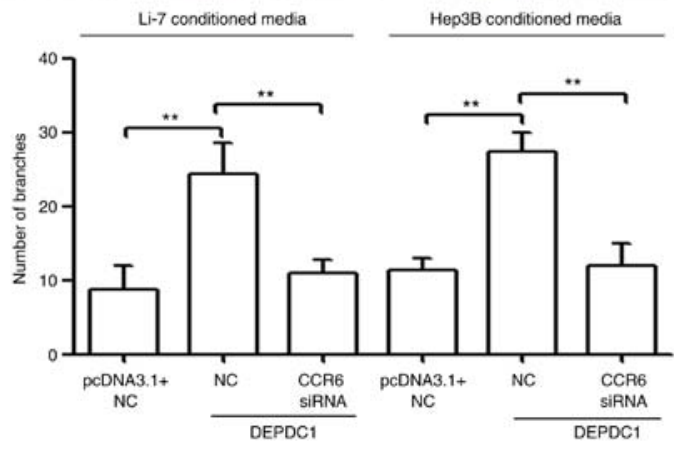

B

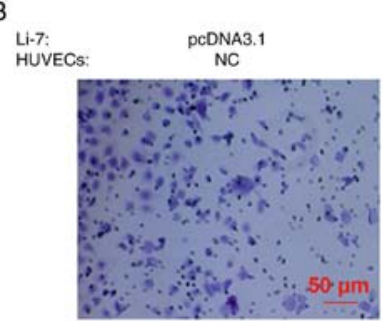

Li-7 conditioned media DEPDC
NC

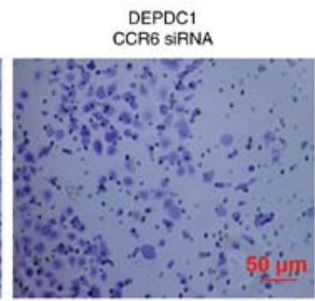

Hep3B:
HUVECs: pCDNA3.1 NC

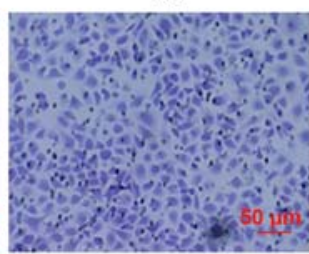

Hep3B conditioned media NC
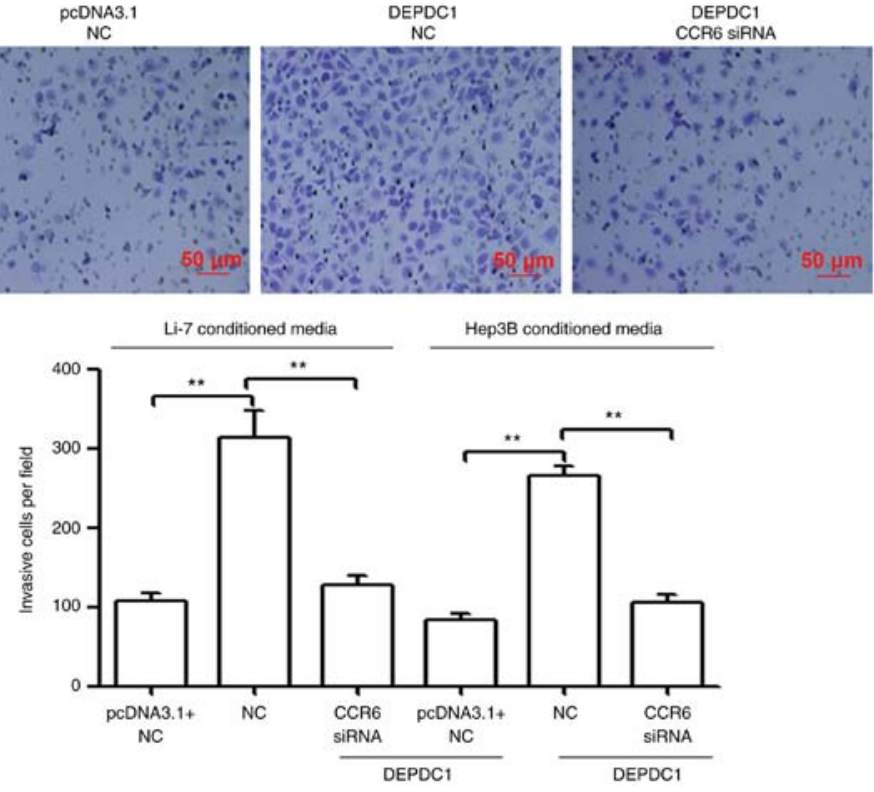

Figure 7. CCR6 knockdown in HUVECs partially reverses the effects of DEPDC1 overexpression on angiogenesis and invasion. (A) TCM was collected from Li-7 and Hep3B cells treated with pcDNA3.1 or DEPDC1 expression vectors. A tube formation assay was performed using HUVECs pretreated with NC or CCR6 siRNA with the indicated conditioned media. Scale bar, $100 \mu \mathrm{m}$. (B) HUVEC cell invasion s was determined via a transwell assay. Each experiment was performed in triplicate. Scale bar, $50 \mu \mathrm{m} .{ }^{* *} \mathrm{P}<0.01$. CCR6, chemokine (C-C motif) receptor 6; HUVECs, human umbilical vein endothelial cells; DEPDC1, DEP domain containing 1; TCM, tumor cell-conditioned medium; NC, negative control; siRNA, small interfering RNA. 


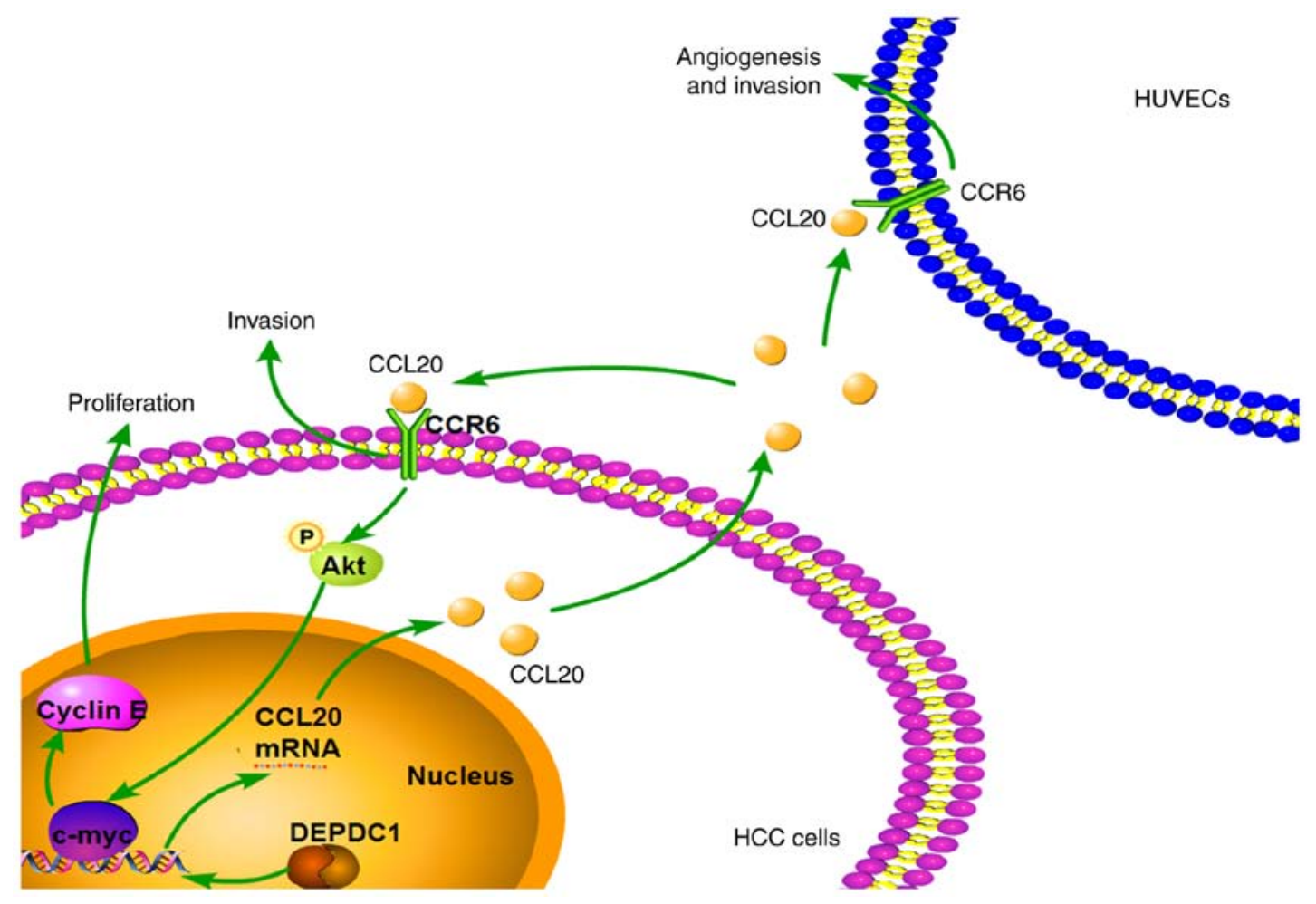

Figure 8. Schematic model depicting the mechanism of DEPDC1-induced HCC progression. DEPDC1 promotes HCC cell proliferation, colony formation and invasion via the CCL20/CCR6 axis. DEPDC1 also induces the activation of p-Akt via the CCL20/CCR6 axis, thereby promoting the protein expression of c-myc and cyclin E1 and HCC cell proliferation. DEPDC1-induced HCC cell-derived CCL20 regulates HUVEC angiogenesis and invasion by mediating endothelial CCR6. DEPDC1, DEP domain containing 1; HCC, hepatocellular carcinoma; CCL20, chemokine (C-C motif) ligand 20; CCR6, chemokine (C-C motif) receptor 6; p-, phosphorylated; HUVEC, human umbilical vein endothelial cells.

and invasion capability of Li-7 and Hep3B cells. DEPDC1 downregulation also significantly inhibited tumourigenesis in xenograft mouse models, which may be due to the inhibition of cell proliferation and colony formation. Consistent with these results, Qu et al (13) demonstrated that DEPDC1 knockdown inhibited HCC cell proliferation, colony formation, migration and invasion. In addition, DEPDC1 silencing via siRNA reduced the viability and invasion capability of prostate cancer cell lines (24), while DEPDC1 upregulation promoted prostate cancer cell proliferation and metastases (25). Collectively, the data indicated that DEPDC1 may serve an important oncogenic role in HCC progression.

To elucidate the underlying molecular mechanism of DEPDC1 in the regulation of HCC progression, gene microarray analysis was performed. GO analysis results revealed that differentially expressed genes induced by DEPDC1 knockdown were enriched in $\mathrm{C}-\mathrm{X}-\mathrm{C}$ chemokine binding, vasculature development, blood vessel development and the extracellular matrix. KEGG pathway analysis revealed that differentially expressed genes induced by DEPDC1 knockdown were significantly enriched in cytokine-cytokine receptor interactions. Gene microarray data also demonstrated that genes associated Cytokine-cytokine receptor interactions, such as CCL20 and CCR6, were significantly downregulated in Huh-7 cells transfected with DEPDC1 siRNA. CCL20 and its physiological sole receptor, CCR6, have been determined to be involved in the proliferation and metastasis of normal cells and tumor cells $(23,26-31)$. For instance, in breast epithelial cells, CCL20/CCR6 increased cell migration via protein kinase C- $\alpha$-activate Src, thereby inducing the activation of Akt, $\mathrm{JNK}$, and NF-kB pathways, and inducing cell proliferation via the ERK1/2/mitogen-activated protein kinase pathway (23). In colorectal cancer cells, CCL20/CCR6 induced cell proliferation and migration by activating of ERK1/2, stress-activated protein kinase/JNK and Akt signaling (26). In addition, CCL20 and CCR6 have been demonstrated to be upregulated in HCC tissue $(15,32)$, and a high expression of CCL20 was associated with poorer survival and recurrence-free survival rates (33). Furthermore, the CCL20/CCR6 axis induced HCC cell proliferation, adhesion and chemotactic migration (14). The CCL20/CCR6 axis also contributes to hepatic angiogenesis in HCV-associated HCC (16). Active angiogenesis and metastasis are responsible for poor patient survival rates and the rapid recurrence of HCC (34). In the present study, RT-qPCR and western blotting revealed that DEPDC1 downregulation significantly suppressed the expression of CCL20 and CCR6 mRNA, which was consistent with gene microarray data. In addition, the results of the present study demonstrated that CCL20 and CCR6 mRNA and protein expression were significantly increased in HCC tissue when compared with matched adjacent normal liver tissue, and were positively correlated with the expression of DEPDC1. These results indicated that the CCL20/CCR6 axis may be an important intermediary of DEPDC1-mediated HCC progression. To verify this hypothesis, CCL20 protein was inhibited via CCL20 siRNA in HCC cells pretreated with the DEPDC1 expression vector. The results revealed that CCL20 knockdown reversed the effects of DEPDC1 overexpression on the cell proliferation, colony 
formation and invasion of HCC cells. Furthermore, CCR6 knockdown via siRNA also rescued the effects of DEPDC1 overexpression on HCC cell proliferation, colony formation and invasion. In normal breast cells, the CCL20/CCR6 axis is known to induce the expression of cell cycle-associated proteins (c-myc, c-Fos and cyclin E1) by activating the PI3K/Akt pathway. The present study revealed that DEPDC1 upregulation induced the expression of p-Akt, c-myc and cyclin E1, while CCL20 or CCR6 knockdown reversed these effects. Thus, the results of the present study indicated that DEPDC1 promoted HCC cell proliferation, colony formation and invasion by regulating the CCL20/CCR6 axis. In addition, the present study demonstrated that DEPDC1 overexpression resulted in the enhanced angiogenesis and invasion of HCC cells. Conversely, CCL20 knockdown was revealed to reverse the effects of DEPDC1 overexpression. It has been previously demonstrated that CCL20 induces HUVEC blood vessel formation and invasion by mediating endothelial CCR6 (16). Thus, the present study hypothesized that endothelial CCR6 may be critical for DEPDC1-CCL20-angiogenesis. To confirm this, CCR6 expression was inhibited in HUVECs. The results revealed that CCR6 knockdown reversed the effects of DEPDC1 overexpression on angiogenesis and invasion of HUVECs. These results indicated that activation of CCL20/CCR6 axis induced by DEPDC1 may be one of the mechanisms that underlie DEPDC1-mediated HCC progression.

In conclusion, the present study confirmed the effect of DEPDC1 in HCC cell proliferation, colony formation and invasion. Furthermore, the results revealed that DEPDC1 promoted HUVEC angiogenesis and invasion. DEPDC1-mediated HCC progression was also determined to be at least partially dependent on the CCL20/CCR6 axis. The results of the present study may provide a new prospective on the role and mechanism of DEPDC1 in HCC.

\section{Acknowledgements}

Not applicable.

\section{Funding}

The present study was supported by the National Natural Science Foundation of China (grant no. 81802398).

\section{Availability of data and materials}

The datasets used and analyzed during the present study are available from the corresponding author on reasonable request.

\section{Authors' contributions}

WG, ZW and SY designed the present study. WG, HLi and HLiu performed the IHC and the RT-qPCR examinations of clinical tissues. HLi and WG collected patient samples and patient information and performed the statistical analysis and the interpretation of the clinical data. WG, HLiu and XM performed the cell experiments and animal experiments and contributed to the statistical analysis and the interpretation of the data. WG wrote the manuscript. ZW and SY reviewed and edited the manuscript. ZW and SY supervised the work of the research group. All authors read and approved the final version of the manuscript.

\section{Ethics approval and consent to participate}

The present study was approved by the Ethical Committee of the First Affiliated Hospital of Chongqing Medical University (Chongqing, China). All patients provided written informed consent.

\section{Patient consent for publication}

Not applicable.

\section{Competing interests}

The authors declare that they have no competing interests.

\section{References}

1. Siegel R, Naishadham D and Jemal A: Cancer statistics, 2013. CA Cancer J Clin 63: 11-30, 2013.

2. Forner A, Llovet JM and Bruix J: Hepatocellular carcinoma. Lancet 379: 1245-1255, 2012.

3. Bellissimo F, Pinzone MR, Cacopardo B and Nunnari G: Diagnostic and therapeutic management of hepatocellular carcinoma. World J Gastroenterol 21: 12003-12021, 2015.

4. Intaraprasong P, Siramolpiwat S and Vilaichone RK: Advances in management of hepatocellular carcinoma. Asian Pac J Cancer Prev 17: 3697-3703, 2016.

5. Bruix J, Gores GJ and Mazzaferro V: Hepatocellular carcinoma: Clinical frontiers and perspectives. Gut 63: 844-855, 2014.

6. Tosi A, Dalla Santa S, Cappuzzello E, Marotta C, Walerich D, Del Sal G, Zanovello P, Sommaggio R and Rosato A: Identification of a HLA-A*0201-restricted immunogenic epitope from the universal tumor antigen DEPDC1. Oncoimmunology 6: e1313371, 2017.

7. Kanehira M, Harada Y, Takata R, Shuin T, Miki T, Fujioka T, Nakamura $\mathrm{Y}$ and Katagiri T: Involvement of upregulation of DEPDC1 (DEP domain containing 1) in bladder carcinogenesis. Oncogene 26: 6448-6455, 2007.

8. Kikuchi R, Sampetrean O, Saya H, Yoshida K and Toda M: Functional analysis of the DEPDC1 oncoantigen in malignant glioma and brain tumor initiating cells. J Neurooncol 133: 297-307, 2017.

9. Feng X, Zhang C, Zhu L, Zhang L, Li H, He L, Mi Y, Wang Y, $\mathrm{Zhu} \mathrm{J}$ and $\mathrm{Bu} \mathrm{Y}$ : DEPDC1 is required for cell cycle progression and motility in nasopharyngeal carcinoma. Oncotarget 8: 63605-63619, 2017.

10. Mi Y, Zhang C, Bu Y, Zhang Y, He L, Li H, Zhu H, Li Y, Lei Y and Zhu J: DEPDC1 is a novel cell cycle related gene that regulates mitotic progression. BMB Rep 48: 413-418, 2015.

11. Yuan SG, Liao WJ, Yang JJ, Huang GJ and Huang ZQ: DEP domain containing 1 is a novel diagnostic marker and prognostic predictor for hepatocellular carcinoma. Asian Pac J Cancer Prev 15: 10917-10922, 2014.

12. Li A, Wang Q, He G, Jin J and Huang G: DEP domain containing 1 suppresses apoptosis via inhibition of A20 expression, which activates the nuclear factor $\kappa \mathrm{B}$ signaling pathway in HepG2 cells. Oncol Lett 16: 949-955, 2018.

13. Qu D, Cui F, Lu D, Yang Y and Xu Y: DEP domain containing 1 predicts prognosis of hepatocellular carcinoma patients and regulates tumor proliferation and metastasis. Cancer Sci 110: 157-165, 2019.

14. Du D, Liu Y, Qian H, Zhang B, Tang X, Zhang T and Liu W: The effects of the CCR6/CCL20 biological axis on the invasion and metastasis of hepatocellular carcinoma. Int J Mol Sci 15: 6441-6452, 2014.

15. Huang F and Geng XP: Chemokines and hepatocellular carcinoma. World J Gastroenterol 16: 1832-1836, 2010. 
16. Benkheil M, Van Haele M, Roskams T, Laporte M, Noppen S, Abbasi K, Delang L, Neyts J and Liekens S: CCL20, a direct-acting pro-angiogenic chemokine induced by hepatitis $\mathrm{C}$ virus (HCV): Potential role in HCV-related liver cancer. Exp Cell Res 372: 168-177, 2018.

17. Berretta M, Rinaldi L, Di Benedetto F, Lleshi A, De Re V, Facchini G, De Paoli P and Di Francia R: Angiogenesis inhibitors for the treatment of hepatocellular carcinoma. Front Pharmaco 7: $428,2016$.

18. Zhu H, Gan X, Jiang X, Diao S, Wu H and Hu J: ALKBH5 inhibited autophagy of epithelial ovarian cancer through miR-7 and BCL-2. J Exp Clin Cancer Res 38: 163, 2019.

19. Livak KJ and Schmittgen TD: Analysis of relative gene expression data using real-time quantitative PCR and the 2(-Delta Delta C(T)) method. Methods 25: 402-408, 2001

20. Chen XY, Wang Q, Gu K, Li A, Fu X, Wang Y, Gu W and Wen Y: Effect of YAP on an immortalized periodontal ligament stem cell line. Stem Cells Int 2019: 6804036, 2019.

21. Fang JH, Zhou HC, Zeng C, Yang J, Liu Y, Huang X, Zhang JP, Guan XY and Zhuang SM: MicroRNA-29b suppresses tumor angiogenesis, invasion, and metastasis by regulating matrix metalloproteinase 2 expression. Hepatology 54: 1729-1740, 2011.

22. Yang C, Xu Y, Cheng F, Hu Y, Yang S, Rao J and Wang X: miR-1301 inhibits hepatocellular carcinoma cell migration, invasion, and angiogenesis by decreasing Wnt/ $\beta$-catenin signaling through targeting BCL9. Cell Death Dis 8: e2999, 2017.

23. Marsigliante S, Vetrugno C and Muscella A: CCL20 induces migration and proliferation on breast epithelial cells. J Cell Physiol 228: 1873-1883, 2013.

24. Ramalho-Carvalho J, Martins JB, Cekaite L, Sveen A, Torres-Ferreira J, Graça I, Costa-Pinheiro P, Eilertsen IA, Antunes L, Oliveira J, et al: Epigenetic disruption of miR-130a promotes prostate cancer by targeting SEC23B and DEPDC1. Cancer Lett 385: 150-159, 2017.

25. Huang L, Chen K, Cai ZP, Chen FC, Shen HY, Zhao WH, Yang SJ, Chen XB, Tang GX and Lin X: DEPDC1 promotes cell proliferation and tumor growth via activation of E2F signaling in prostate cancer. Biochem Biophys Res Commun 490: 707-712, 2017.
26. Brand S, Olszak T, Beigel F, Diebold J, Otte JM, Eichhorst ST, Göke B and Dambacher J: Cell differentiation dependent expressed CCR6 mediates ERK-1/2, SAPK/JNK, and Akt signaling resulting in proliferation and migration of colorectal cancer cells. J Cell Biochem 97: 709-723, 2006.

27. Frick VO, Rubie C, Keilholz U and Ghadjar P: Chemokine/chemokine receptor pair CCL20/CCR6 in human colorectal malignancy: An overview. World J Gastroenterol 22: 833-841, 2016.

28. Zhang XP, Hu ZJ, Meng AH, Duan GC, Zhao QT and Yang J: Role of CCL20/CCR6 and the ERK signaling pathway in lung adenocarcinoma. Oncol Lett 14: 8183-8189, 2017.

29. Han G, Wu D, Yang Y, Li Z, Zhang J and Li C: CrkL meditates CCL20/CCR6-induced EMT in gastric cancer. Cytokine 76: 163-169, 2015.

30. Ghadjar P, Rubie C, Aebersold DM and Keilholz U: The chemokine CCL20 and its receptor CCR6 in human malignancy with focus on colorectal cancer. Int J Cancer 125: 741-745, 2009.

31. Nandi B, Pai C, Huang Q, Prabhala RH, Munshi NC and Gold JS: CCR6, the sole receptor for the chemokine CCL20, promotes spontaneous intestinal tumorigenesis. PLoS One 9: e97566, 2014.

32. Rubie C, Frick VO, Wagner M, Rau B, Weber C, Kruse B, Kempf K, Tilton B, König J and Schilling M: Enhanced expression and clinical significance of CC-chemokine MIP-3 alpha in hepatocellular carcinoma. Scand J Immunol 63: 468-477, 2006.

33. Hou KZ, Fu ZQ and Gong H: Chemokine ligand 20 enhances progression of hepatocellular carcinoma via epithelial-mesenchymal transition. World J Gastroenterol 21: 475-483, 2015.

34. Fernandez M, Semela D, Bruix J, Colle I, Pinzani M and Bosch J: Angiogenesis in liver disease. J Hepatol 50: 604-620, 2009.

This work is licensed under a Creative Commons Attribution-NonCommercial-NoDerivatives 4.0 International (CC BY-NC-ND 4.0) License. 